\title{
Dual-plane PIV investigation of acoustically excited jets in a swirl nozzle
}

\author{
Gavita S. Regunath ${ }^{1}$, William B. Zimmerman ${ }^{2}$, and Julia M. Rees ${ }^{3}$ \\ ${ }^{1}$ Department of Aeronautical and Automotive Engineering, Stewart Miller Building, Loughborough University, \\ Leicestershire, LE11 3TU, UK \\ ${ }^{2}$ Department of Chemical and Biological Engineering, The University of Sheffield, Mappin Street, Sheffield, S1 3JD, UK \\ ${ }^{3}$ School of Mathematics and Statistics, The University of Sheffield, Hicks Building, Hounsfield Road, Sheffield, S3 7RH, UK
}

Correspondence to: Julia M. Rees (j.rees@sheffield.ac.uk)

Received: 23 June 2015 - Published in Nonlin. Processes Geophys. Discuss.: 4 September 2015

Revised: 16 January 2016 - Accepted: 10 March 2016 - Published: 4 April 2016

\begin{abstract}
A novel dual-plane dye laser particle image velocimetry (PIV) technique used to analyze helicity and energy dissipation in an unexcited turbulent swirling jet of pressurized cold air has established that regions within the flow field of the jet exhibiting high helicity are correlated regions of high turbulent kinetic energy dissipation. This PIV configuration provides estimates of all components of the velocity gradient tensor, facilitating calculation of the helicity from the vorticity components. Application of this novel dual-plane PIV technique is extended in this study to investigate helical structures in a turbulent swirling jet where the underlying shear flow is subjected to external acoustic sinusoidal forcing in a plane perpendicular to the central axis of the jet. It was found that acoustic excitation had a significant effect on the mean velocity profile parallel to the direction of the jet. The horizontal forcing resulted in the generation of vorticity that was skewed with a pitch that favored a distribution of angles around $90^{\circ}$ with respect to the velocity vector. The distribution of the time-averaged helicity angle indicated organized helical activity, but such activity is not dominated by large-scale coherent structures of maximal helicity.
\end{abstract}

\section{Introduction}

Helical structures are a common phenomenon in geophysical flows, for example, in magnetohydrodynamics (Moffatt, 1978), storm systems (Lilly, 1986), underwater gravity currents (Sumner et al., 2014), and atmospheric inertia-gravity waves (Viúdez and Dritschel, 2006). It is well established that helical coherent structures play a crucial role in turbulent flows. However, the role of helicity in three-dimensional turbulent flows is still enigmatic. Complex interactions can occur between different scales of the motion. In addition, nonlinearity and intermittency influence the scaling laws.

One of the main effects of helicity in neutral isotropic turbulence is the inhibition of energy transfer at large eddy sizes. Kraichnan (1973) investigated analytically the interaction of two helical waves, concluding that the presence of a non-zero mean helicity in a turbulent flow inhibits the energy transfer to small scales. This was confirmed numerically by André and Lesieur (1977). Thus homogeneous turbulence with significant mean helicity exhibits a much slower rate of decay of turbulence and its corresponding energy than its zero-mean helicity counterpart.

When rotational effects are present, significant differences exist between helical and non-helical turbulent flows (Pouquet and Mininni, 2010). Orlandi (1997) carried out direct numerical investigations of helicity fluctuations and the production of turbulent energy in rotating and non-rotating pipes. Results showed that helical dynamics were important across all scales and that there existed a correlation between maximal helicity and low energy dissipation. Such an occurrence is an attractive prospect as it implies that effective dispersion of heat or mass using low mechanical energy consumption is possible with the use of helical flows with maximal helicity, that is, via helical coherent structures. In contrast, three-dimensional laminar studies of flow through a staggered herringbone microchannel at low Reynolds number conducted by Hassell and Zimmerman (2006) revealed 
that an increase in helicity in flows corresponded to an increase in the energy dissipation.

Khomenko et al. (1991) showed that for the case of compressible turbulence, large-scale helical structures can be generated from small-scale turbulent helices. Subsequently, Khomenko and Zimmerman (1994) demonstrated the existence of an inverse cascade of helical structure formation, where large helical structures are formed by small helical structures. Exploitation of this inverse cascade can lead to improved mixing efficiencies due to the dispersion effects of helical coherent structures. In turbulent flows, Zimmerman (1996a) demonstrated that intensified mixing could be achieved with helical coherent structures. Simulations of mixing showed up to a 20 -fold improvement in eddy diffusivities compared to that possible from an equivalent input of energy to base state of isotropic turbulence (Zimmerman, 1996b). This was attributed to the three-dimensional dispersive property of helical flows, which enhances molecular diffusion due to the creation of strong gradients.

It is well known that two types of instabilities may be exhibited in the shear layer of an unexcited, free jet: the column instability, which causes the whole jet to oscillate as if it were an oscillating elastic column, and the shear layer instability, which induces unstable waves in the mixing layer that surrounds the jet core immediately downstream of the nozzle exit. However, as the shear layer instability progresses downstream, it grows and merges, eventually becoming difficult to distinguish from the column instability. The distinguishing feature between these two types of instabilities is their dependence on the resonant frequency at the nozzle exit Reynolds number, Re. This is determined by the nondimensional Strouhal number, $S t$, given by $S t=\frac{f D}{U_{\mathrm{e}}}$, where $f$ denotes frequency, $D$ is the nozzle exit diameter, and $U_{\mathrm{e}}$ is the velocity at the nozzle exit. It has been found that $S t$ lies between 0.2 and 0.5 for column instability, whilst for shear layer instability the proportionality factor of $\frac{S t}{\sqrt{R e}}$ is 0.012 to 0.017 (Crow and Champagne, 1971; Drobniak and Klajny, 2002). The degree of swirl in a flow is characterized by the non-dimensional swirl number, $S$, given by

$$
S=\frac{\int_{0}^{R} u w r^{2} \mathrm{~d} r}{R \int_{0}^{R} u^{2} r \mathrm{~d} r},
$$

where $R$ is the radius of the nozzle, $u$ and $w$ denote the axial and tangential velocity components, and $r$ is the radial coordinate (Sheen et al., 1996).

Regunath et al. (2008) reported on the use of a novel dualplane dye particle image velocimetry (PIV) laser system for investigating energy dissipation and helicity in a turbulent swirling flow. The PIV system comprised a single-frequency Nd:YAG laser that generated two parallel light sheet planes of different wavelengths. This was operated in conjunction with three CCD cameras. Results from this study showed that in an unforced turbulent swirling jet, high rates of turbulent kinetic energy dissipation correlated with regions exhibiting strong helicity. The purpose of this paper is to extend the work of Regunath et al. (2008) by investigating the role of helicity in a turbulent swirling forced jet. Here, the term "forced jet" implies the use of an external force to perturb the shear layer in order to generate helical waves. By applying sinusoidal perturbations in the form of acoustic waves, helical structures are formed. Further understanding of the interplay between helicity and turbulence in this setting will impact on our knowledge of helicity-turbulence interactions in a range of geophysical situations.

The experimental setup, together with a brief overview of the methodology of the PIV technique, is given in Sect. 2. Results from the dual-plane PIV analysis of acoustically excited jets are presented in Sect. 3 and conclusions are summarized in Sect. 4.

\section{Experimental setup and statistical analysis of PIV data}

PIV enables the instantaneous velocity field of a flow to be recorded by means of a technique based upon crosscorrelations. Originally used to obtain two-dimensional velocity fields, developments over the last decade have led to the successful realization of three-dimensional velocity fields (Lynch and Thurow, 2011). Statistical properties of the mean and turbulent components can be estimated from ensemble averaging (Ullum et al., 1998). However, accuracy of the statistical analysis depends on the sample size over which the ensemble averages are formed (Uzol and Camci, 2001). Depending on the degree of variability of the flow, the number of image pairs required to provide the desired level of statistical accuracy typically ranges from 200 to 750 . A stereoscopic, i.e., three-dimensional, PIV technique used in conjunction with a dual-plane dye laser system can be used to estimate all nine velocity gradient components, $\frac{\partial u_{i}}{\partial x_{j}}$, thus facilitating the determination of all three vorticity components, $\omega_{x}, \omega_{y}$, and $\omega_{z}$. The experimental configuration used is described in detail in Regunath et al. (2008). In the current study the experimental apparatus is enhanced by a system of eight loudspeakers deployed so as to induce sinusoidal perturbations in the form of acoustic waves (Fig. 1). These sinusoidal perturbations give rise to a forced jet.

\section{Experimental design}

Full details of the basic experimental configuration are given in Regunath et al. (2008), so only a brief outline will be given here. A centrifugal compressor supplied the working fluid of pressurized cold air through a twisted vane swirl nozzle to impart both a tangential and an axial momentum within the flow field, thus giving rise to a swirling motion (Fig. 2). Water droplets generated by an ultrasonic hu- 


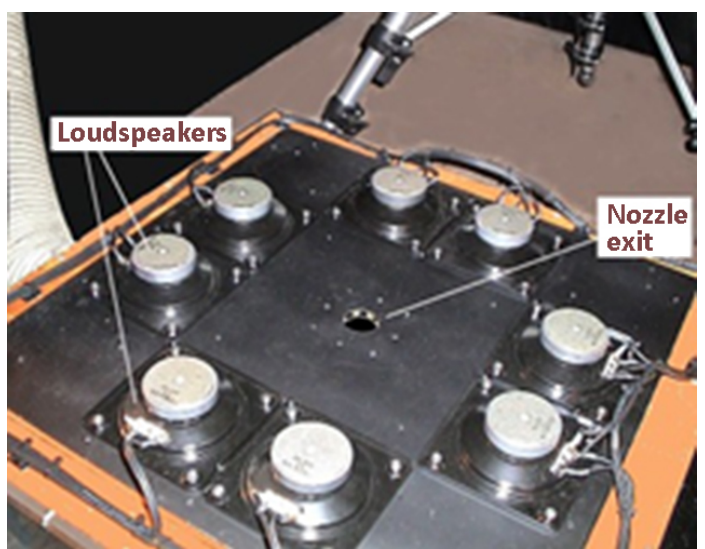

Figure 1. Photographic image of the acoustic excitation chamber with eight loudspeakers that are placed in a flatwise manner to generate the necessary acoustic pressure in order to perturb the jet shear layer at the nozzle exit.

midifier were used as tracer particles. The exit diameter, $D$, of the nozzle had a width of $32 \mathrm{~mm}$. Dual-plane dye laser PIV measurements were made in the near-jet region, $0<y / D<1$, where $y$ denotes the streamwise direction. Vertical light sheets in two planes slightly displaced from one another were used to illuminate the flow field. The center of the green light sheet plane, $\lambda=532 \mathrm{~nm}$, was aligned at the centerline of the jet $(z=0 \mathrm{~mm})$, whilst the center of the yellow light sheet plane, $\lambda=572 \mathrm{~nm}$, was positioned along the plane $z=-1 \mathrm{~mm}$ (Fig. 3). The thickness of each light sheet was measured to be $2 \mathrm{~mm}$; thus, the yellow light sheet extended from $z=-2 \mathrm{~mm}$ to $z=0 \mathrm{~mm}$ and the green light sheet extended from $z=-1 \mathrm{~mm}$ to $z=1 \mathrm{~mm}$. Although the light sheets overlapped, the optical filters that were placed in front of the cameras only permitted one of the two wavelengths, $\lambda=532 \mathrm{~nm}$ or $\lambda=572 \mathrm{~nm}$, to be captured. The dual-plane dye laser PIV system was used to obtain stereoscopic measurements on the green light sheet. Planar two-dimensional measurements were made on the yellow light sheet. Ensemble time averages based on 1000 PIV image pairs of the instantaneous velocity vectors were formed to produce the mean velocity field within each measured plane. The PIV system was limited to the frequency of cameras, which was $4 \mathrm{~Hz}$. Therefore 1000 image pairs were recorded at a frequency of $4 \mathrm{~Hz}$ over a period of approximately $250 \mathrm{~s}$.

In order to generate the acoustic perturbations required for the present study, an acoustic excitation chamber comprising eight loudspeakers was arranged circumferentially around the jet nozzle exit. Helical acoustic waves were generated via inputted sinusoidal signals, of frequency $f$, that were phase shifted by $\frac{\pi}{2}$ between each adjacent speaker, resulting in a sequential pressure variation at the nozzle exit. A four-channel high-performance power amplifier with a maximum power of $4 \times 120 \mathrm{~W}$ was used to amplify the generated sinusoidal signals. A trigger signal connected to the PIV synchronizer

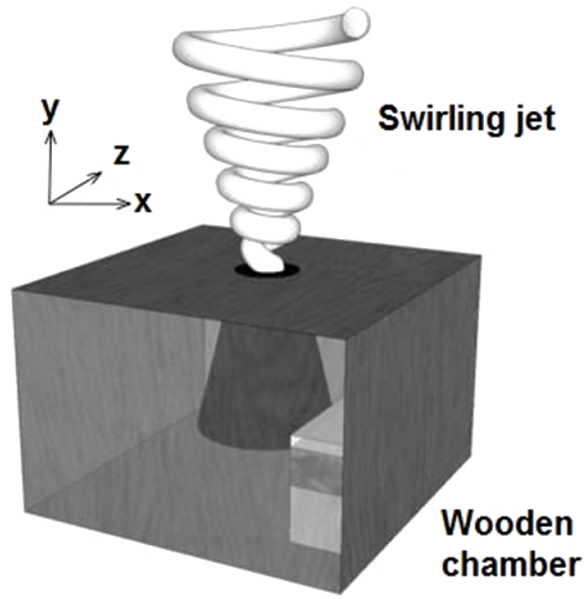

Figure 2. Schematic of the experimental setup. The swirl nozzle is located within the wooden chamber.

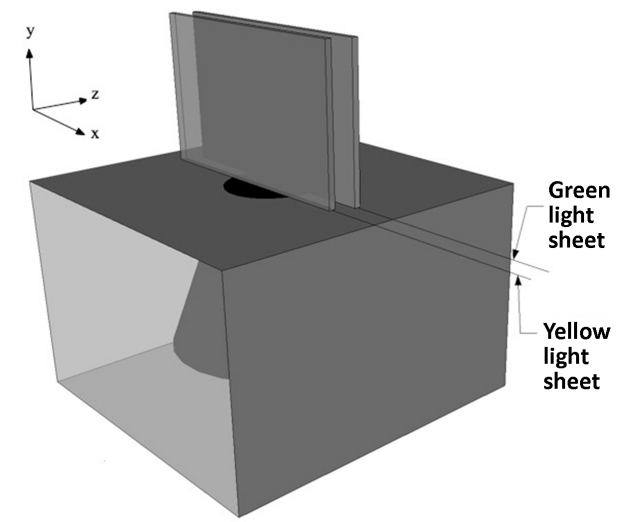

Figure 3. Schematic of the swirling jet chamber showing the orientation and position of the two light sheets.

enabled the PIV system to capture images at appropriate time intervals. Measurements were made at frequencies of 200, 400 , and $600 \mathrm{~Hz}$ to investigate the effectiveness of input frequency for generating helical structures in the resultant flow field. These frequencies correspond to Strouhal numbers, $S t$, of $0.93,1.86$, and 2.80 , respectively. A control experiment without acoustic excitation was performed for comparison purposes. The measured Reynolds number and Swirl number for these experimental investigations were kept constant at $R e=12890$ and $S=0.43$.

\section{Results}

The dual-plane dye laser PIV technique was used to illuminate the vertical planes aligned along $z=-1 \mathrm{~mm}$ and $z=0 \mathrm{~mm}$ that permitted measurements of the velocity vector field to be made. The single camera vector field constructed from measurements obtained from the plane $z=-1$ 


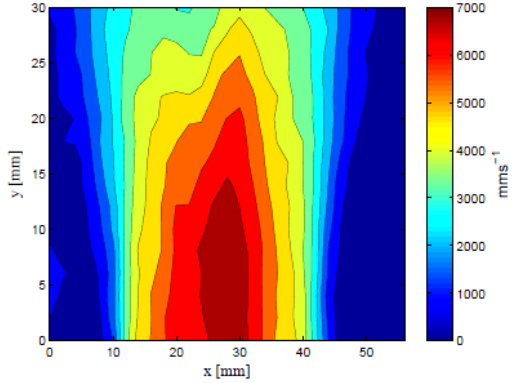

(a) Unexcited jet

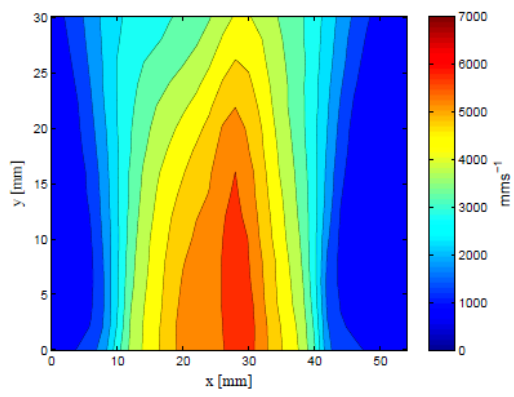

(c) Excited jet, $S t=01.86$

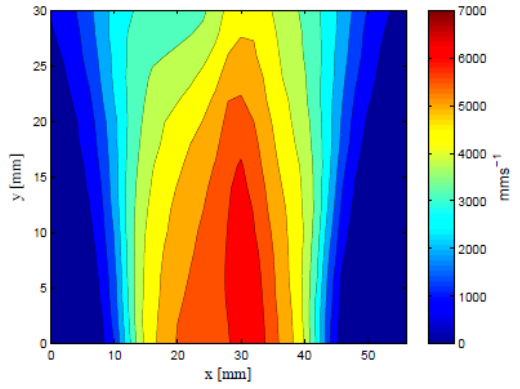

(b) Excited jet, $S t=0.93$

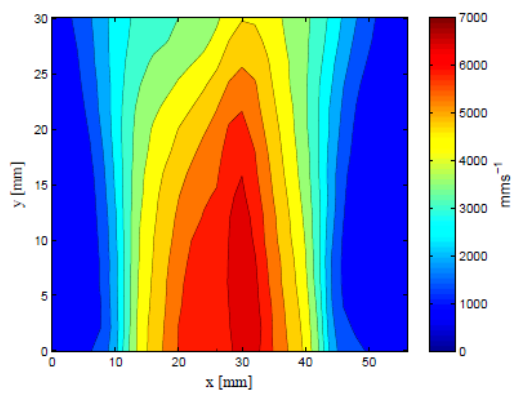

(d) Excited jet, $S t=2.80$

Figure 4. Magnitude of the velocity field on the $z=0 \mathrm{~mm}$ plane: (a) for an unexcited jet; (b) for an excited jet with $S t=0.93$; (c) for an excited jet with $S t=1.86$; and (d) for an excited jet with $S t=2.80$.

was used in conjunction with the stereoscopic vector field from the $z=0 \mathrm{~mm}$ plane to compute all nine velocity gradients in the $z=0 \mathrm{~mm}$ plane. The velocity gradient fields were obtained from second-order central differences of the measured velocity component values within each plane and from first-order forward differences between the two illuminated planes aligned parallel to the $y$ axis (Fig. 3).

Contours of the magnitude of the mean velocity field on the plane $z=0 \mathrm{~mm}$ for an unforced jet and for forced jets for a range of Strouhal numbers $(S t=0.93,1.86$, and 2.80) are presented in Fig. 4. To facilitate comparisons, uniform color scaling has been used in each sub-figure. The jet structure in each case is qualitatively similar, although a larger degree of irregularity is noticed in the instance of the unexcited jet and the magnitude of the velocity field at the nozzle exit is greater.

To further investigate the effect of excitation on the swirling jet, the decay of the time-mean axial velocity along the jet centerline is compared for excited and unexcited cases in Fig. 5. For these measurements, the light sheets were placed parallel to the $y$ axis, so that the jet centreline is measurable. The results show the average axial velocities along the jet centerline axis for the case of an unexcited jet and for excited jets at different $S t$ numbers. The velocities of the excited jets at the nozzle exit were found to be 15 to $17 \%$ lower than the exit velocity of the unexcited jet. After a slight increase in mean axial velocity in the vicinity of the nozzle exit, the axial velocities of the excited jets then decreased mono-

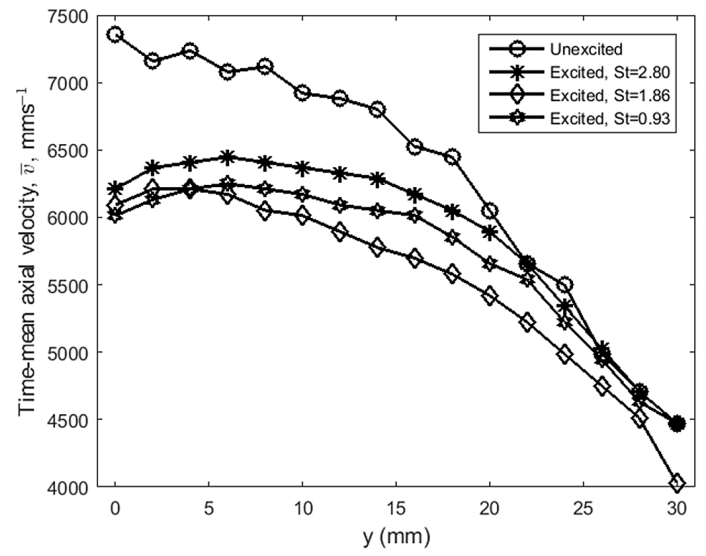

Figure 5. Decay of time-mean axial velocities along the jet centerline axis for the case of an unexcited jet and for excited jets at different $S t$ numbers.

tonically. For example, the axial velocities of the excited jets at $S t=2.80(f=600 \mathrm{~Hz})$ and $S t=0.93(f=200 \mathrm{~Hz})$ show that the mean axial velocity increases across the first $6 \mathrm{~mm}$ downstream of the exit nozzle. The axial velocity of the excited jet with $S t=1.86(f=400 \mathrm{~Hz})$ increases over the first $2 \mathrm{~mm}$, then decreases along the jet centerline. We can attribute this behavior of the velocity profiles as a response to the perturbations applied to the jet at the nozzle exit since for the case of the unexcited jet the maximal velocity was 


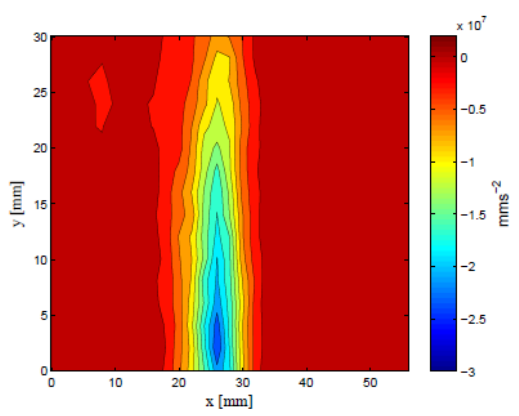

(a) Unexcited jet

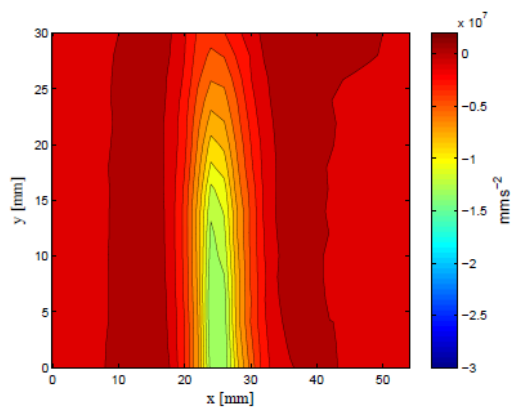

(c) Excited jet, $S t=1.86$

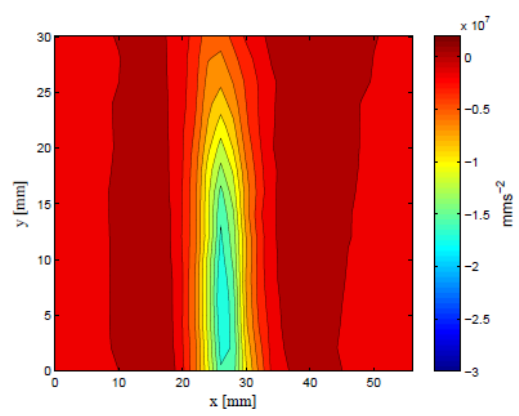

(b) Excited jet, $S t=0.93$

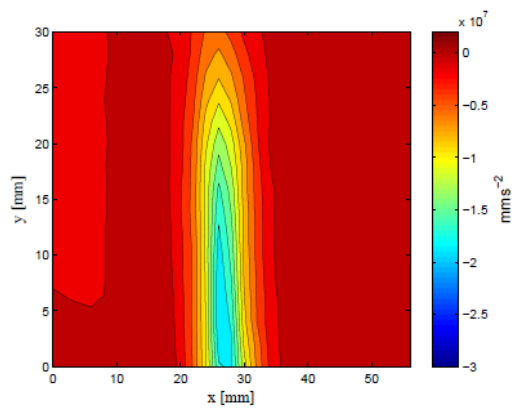

(d) Excited jet, $S t=2.80$

Figure 6. Average helicity density, $\bar{h}$, on the $z=0 \mathrm{~mm}$ plane: (a) for an unexcited jet; (b) for an excited jet with $S t=0.93$; (c) for an excited jet with $S t=1.86$; and (d) for an excited jet with $S t=2.80$.

found to occur at the exit itself, rather than at a downstream location.

The dual-plane dye laser PIV technique permits us to compute all the velocity and vorticity components over the $z=0$ plane; thus, we can calculate the helicity density, $h$, which is defined as the scalar product of the local velocity vector, $\boldsymbol{u}$, and the local vorticity vector, $\boldsymbol{\omega}$, i.e., $h=\boldsymbol{u} \cdot \boldsymbol{\omega}$. The relative uncertainties in the vorticity components $\omega_{x}, \omega_{y}$, and $\omega_{z}$ were 19,16 , and $6 \%$, respectively. For helicity, the relative uncertainty was found to be approximately $13 \%$. We are also able to determine the helicity angle, $\theta$, from

$\cos \theta=\frac{\boldsymbol{u} \cdot \boldsymbol{\omega}}{|\boldsymbol{u}||\boldsymbol{\omega}|}$.

Figure 6 shows the computed mean helicity density, $\bar{h}$, for the unexcited and excited jets, respectively. It can be seen that the helicity density values at the nozzle exit have predominantly negative values, which, since helicity density is a pseudoscalar quantity, is indicative of clockwise rotation (left-hand chirality). The color scales for these figures represent the helicity density and have been plotted on a uniform scale of $-3 \times 107 \mathrm{~mm} \mathrm{~s}^{-2}$ to $2 \times 106 \mathrm{~mm} \mathrm{~s}^{-2}$. It is observed that the largest magnitude of the average helicity density is associated with the unexcited jet at the nozzle exit.

Figure 7 shows plots of the average helicity angle, $\bar{\theta}$, for the unexcited and excited jets across the $z=0$ plane. Plots of $\bar{\theta}$ provide insight into the occurrence of helical coherent structures (HCS). HCS are structures that have maximal he- licity. These are defined as those where the angle between velocity, $\boldsymbol{u}$, and vorticity, $\boldsymbol{\omega}$, is either $0^{\circ}$ (indicated by blue) or $180^{\circ}$ (indicated by red).

Figure 7 shows the occurrence of a few regions where the helicity angle is close to $0^{\circ}$ (colored dark blue), and some (colored dark red) that indicate a helicity angle around $180^{\circ}$. A bar chart logging the occurrence of the maximal helicity angle for the $z=0$ plane is presented in Fig. 8. The histograms of the unexcited and excited jets all show that there are no counts of a helicity angle of $0^{\circ}$. However, for the excited jet at $S t=1.86$ an occurrence of a helicity angle in the range $180^{\circ} \pm 5^{\circ}$ is logged. Upon further inspection it was found that this angle was in fact $178.5^{\circ}$, which is not significantly different from $180^{\circ}$ due to limitations on the accuracy of measurements. In fact, the modal helicity angle was found to be around $90^{\circ}$.

\section{Conclusions}

Preliminary experimental results of acoustic excitation on swirling jets have been reported. A twisted vane swirling jet was used to generate the swirling flow fields. An acoustic excitation method comprising eight loudspeakers that operated in pairs was used to generate sinusoidal signals to excite the swirling jets by perturbing the shear layer. To measure the effect of acoustic excitation on the helical properties of the resultant flow field, the novel dual-plane dye laser 


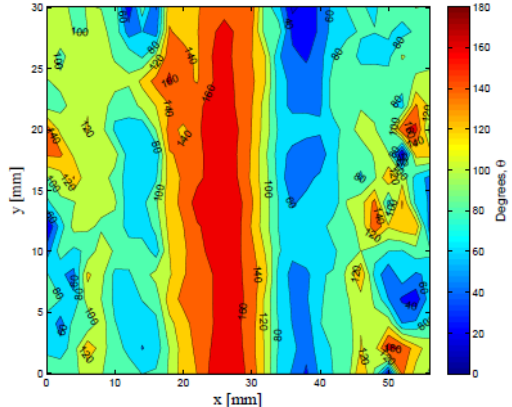

(a) Unexcited jet

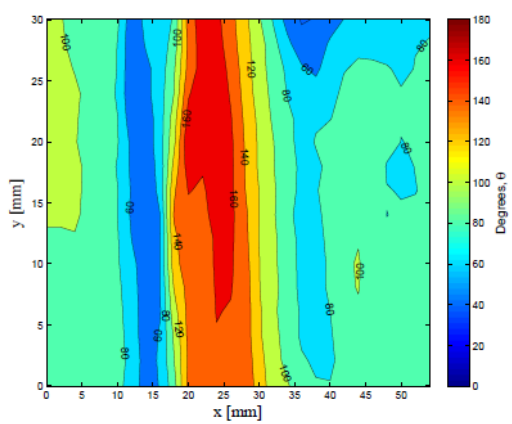

(c) Excited jet, $S t=1.86$

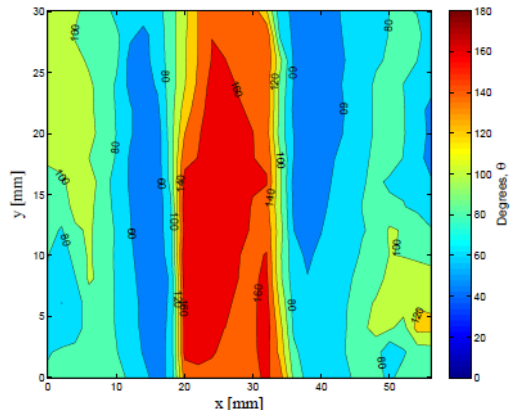

(b) Excited jet, $S t=0.93$

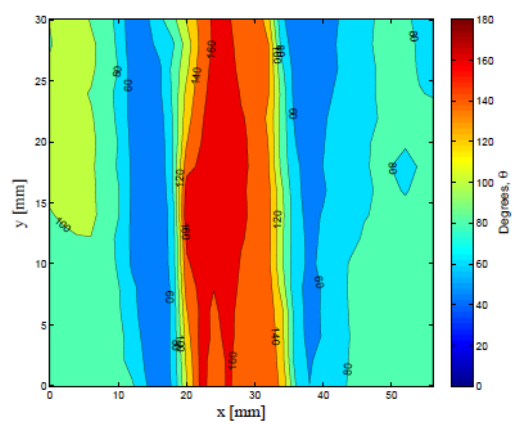

(d) Excited jet, $S t=2.80$

Figure 7. Average helicity angle, $\bar{\theta}$, on the $z=0 \mathrm{~mm}$ plane: (a) for an unexcited jet; (b) for an excited jet with $S t=0.93$; (c) for an excited jet with $S t=1.86$; and (d) for an excited jet with $S t=2.80$.

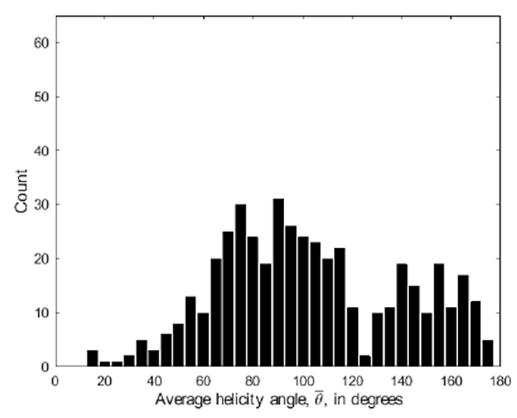

(a)

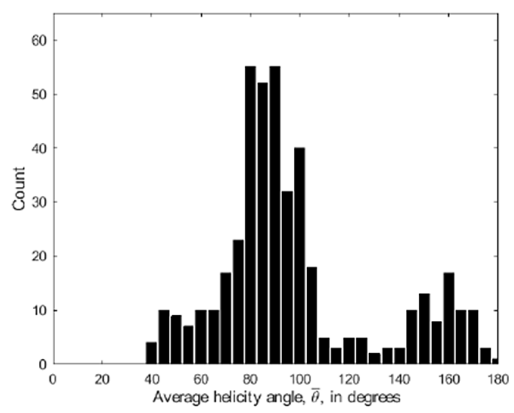

(c)

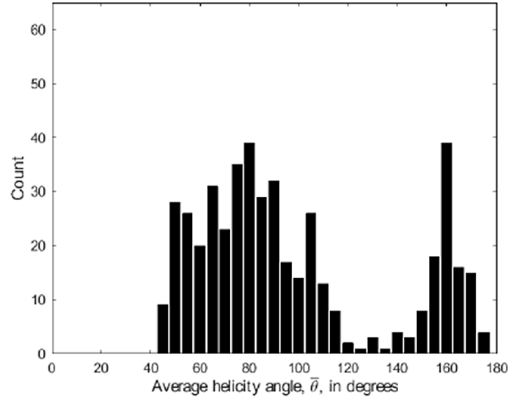

(b)

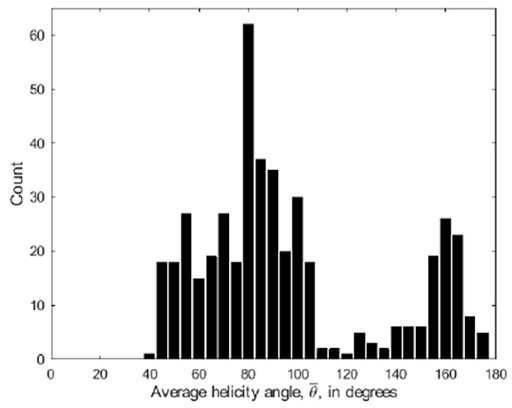

(d)

Figure 8. Bar charts of the average helicity angle, $\bar{\theta}$, in degrees, on the $z=0 \mathrm{~mm}$ plane: (a) for an unexcited jet; (b) for an excited jet with $S t=0.93$; (c) for an excited jet with $S t=1.86$; and (d) for an excited jet with $S t=2.80$. 
PIV method was used. This technique was used to illuminate the $z=-1 \mathrm{~mm}$ and $z=0 \mathrm{~mm}$ vertical planes to measure the fluid velocities. Three different experiments were carried out at frequencies of 200,400 , and $600 \mathrm{~Hz}$, corresponding to Strouhal numbers of $S t=0.93, S t=1.86$, and $S t=2.80$. A further experiment without the effect of acoustic excitation was also conducted to allow comparison of results for excited and unexcited jets. By observing the plots of velocity magnitude, helicity density, and helicity angle, it was found that the jets under acoustic excitation all had different velocity profiles when compared to the jet without any acoustic excitation. Furthermore, the distributions of the average helicity angle, $\bar{\theta}$, revealed a greater degree of skewness between the velocity and vorticity vectors than for the unforced case. Evidence for the existence of measurable organized helical structures was found, though the helical vortices were not dominated by coherent structures exhibiting maximal helicity in either unexcited or excited swirling jets. However, some coherent structures with close to maximal helicity were found to occur.

Acknowledgements. W. B. Zimmerman acknowledges EPSRC grant GR/S08695/01.

Edited by: J. M. Redondo

Reviewed by: two anonymous referees

\section{References}

André, J. C. and Lesieur, M.: Influence of helicity on the evolution of isotropic turbulence at high Reynolds number, J. Fluid Mech., 81, 187-207, 1977.

Crow, S. C. and Champagne, F. H.: Orderly structure in jet turbulence, J. Fluid Mech., 48, 547-591, 1971.

Drobniak, S. and Klajny, R.: Coherent structures of free acoustically stimulated jet, J. Turbulence, 3, 1, 2002.

Hassell, D. G. and Zimmerman, W. B.: Investigation of the convective motion through a staggered herringbone micromixer at low Reynolds number flow, Chem. Eng. Sci., 61, 2977-2985, 2006.

Khomenko, G. A. and Zimmerman, W. B.: Large scale structure evolution and mixing due to small scale helical forcing in a compressible viscous fluid, in: Mixing in Geophysical Flows, edited by: Redondo, J. and Metais, O., UPC Barcelona Press, Barcelona, 233-248, 1994.

Khomenko, G. A., Moiseev, S. S. and Tur, A. V.: The hydrodynamic alphaeffect in a compressible medium, J. Fluid Mech., 225, 355369,1991
Kraichnan, R. H.: Helical turbulence and absolute equilibrium, J. Fluid Mech., 59, 745-752, 1973.

Lilly, D. K.: The structure, energetics and propagation of rotating convective storms. Part I: Energy exchange with the mean flow, J. Atmos. Sci., 43, 113-125, 1986.

Lynch, K. and Thurow, B.: Preliminary development of a 3-D, $3-$ C PIV technique using light field imaging, 29th AIAA Aerodynamic Measurement Technology and Ground Testing Conference, AIAA, Honolulu, HI, June 2011.

Moffatt, H. K.: Magnetic field generation in electrically conducting fields, Cambridge University Press, 343 pp., 1985.

Orlandi, P.: Helicity fluctuations and turbulent energy production in rotating and non-rotating pipes, Phys. Fluids, 9, 2045-2056, 1997.

Pouquet A. and Mininni P. D.: The interplay between helicity and rotation in turbulence: implications for scaling laws and small-scale dynamics, Philos. Trans. A, 368, 1635-1662, doi:10.1098/rsta.2009.0284, 2010.

Regunath, G. S., Zimmerman, W. B., Tesar, V., Hewakandamby, B. N.: Experimental investigation of helicity in turbulent swirling jet using dual-plane dye laser PIV technique, Exp. Fluids, 45, 973-986, doi:10.1007/s00348-008-0515-3, 2008.

Sheen, H. S., Chen, W. J., Jeng, S. Y., and Huang, T. L.: Correction of swirl number for radial-type swirl generator, Exp. Therm. Fluid Sci., 12, 444-451, 1996.

Sumner, E. J., Peakall, J., Dorrell, R. M., Parsons, D. R., Darby, S. E., Wynn, R. B., McPhail, S. D., Perrett, J., Webb, A., and White, D.: Driven around the bend: Spatial evolution and controls on the orientation of helical bend flow in a natural submarine gravity current, J. Geophys. Res.-Oceans, 119, 898-913, doi:10.1002/2013JC009008, 2014.

Ullum, U., Schmidt, J. J., Larsen, P. S., and McCluskey, D. R.: Statistical analysis and accuracy of PIV data, J. Visualization, 1, 205-216, 1998.

Uzol, O. and Camci, C.: The effect of sample size, turbulence intensity and the velocity foeld on the experimental accuracy of ensemle averaged PIV measurements, paper number 1096, 4th International Symposium on Particle Image Velocimetry, 17 19 Sepember 2001, Göttingen Germany, 2001.

Viúdez, A. and Dritschel, D. G.: Spontaneous generation of intertiagravity wave packets by balanced geophysical flows, J. Fluid Mech., 553, 107-117, doi:10.1017/S0022112005008311, 2006.

Zimmerman, W. B.: Fluctuations in passive scalar concentration convected by a helical flow in a conducting fluid, Phys. Fluids, 8 , 1631-1642, 1996a.

Zimmerman, W. B.: Fluctuations in passive tracers due to mixing by coherent structures in isotropic, homogeneous, helical turbulence, IChemE Symposium Series, edited by: Mixing, V. and Benkreira, H., The Institute of Chemical Engineers, Rugby, UK, 213-224, 1996b. 